NBI-HE-97-04

EPHOU-97-002

hep-th/9702019

February 1997

\title{
Non-critical open strings beyond the semi-classical approximation
}

\author{
J. Ambjørn円 \\ The Niels Bohr Institute \\ Blegdamsvej 17, DK-2100 Copenhagen Ø, Denmark, \\ K. Hayasaka[ and R. Nakayama[ \\ Division of Physics, Graduate School of Science, \\ Hokkaido University, Sapporo 060, Japan
}

\begin{abstract}
We studied the lowest order quantum corrections to the macroscopic wave functions $\Gamma(A, \ell)$ of non-critical string theory using the semi-classical expansion of Liouville theory. By carefully taking the perimeter constraint into account we obtained a new type of boundary condition for the Liouville field which is compatible with the reparametrization invariance of the boundary and which is not only a mixture of Dirichlet and Neumann types but also involves an integral of an exponential of the Liouville field along the boundary. This condition contains an unknown function of $A / \ell^{2}$. We determined this function by computing part of the one-loop corrections to $\Gamma(A, \ell)$.
\end{abstract}

\footnotetext{
${ }^{1}$ email ambjorn@nbivms.nbi.dk

2 email hayasaka@particle.phys.hokudai.ac.jp

${ }^{3}$ email nakayama@particle.phys.hokudai.ac.jp
} 


\section{Introduction}

The discretized approach to two-dimensional quantum gravity has been very successful. The underlying lattice regularization, known as dynamical triangulation, provides a rigorous definition of two-dimensional quantum gravity, as well as two-dimensional quantum gravity coupled to a variety of matter fields [1, 2, 3]. Somewhat surprising, it seems easier to perform analytic calculations in this formalism than in the corresponding continuum formalism, i.e. Liouville theory coupled to matter fields. The success of KPZ[ [4] and $D D K[5]$ in calculating the critical exponents of the conformal field theories coupled to two-dimensional quantum gravity has over-shaded the fact that these quantities are the only ones with a physical interpretation which can be calculated in the context of Liouville theory. Actually it has been possible to calculate 2, 3 and 4-point functions [6] and there is also an attempt to obtain two-loop amplitudes in the proper-time gauge [0]. However, extension to general loop amplitudes has not been achieved. Contrary, in the discretized approach it has been possible to calculate macroscopic loop functions [8, 9, 10] as well as so-called two-point functions depending on invariant distances [11, 12]. The various techniques used in the discretized approach, such as matrix models calculations, loop equations or transfer matrices are all of combinatorial nature, and the power of these methods reflects that the solution of discretized two-dimensional quantum gravity is of purely combinatorial nature, maybe due to the lack of gravitons. The semi-classical limit of Liouville theory combined with $K P Z$ scaling has taught us many of the same lessons as the discretized approach, again indicating the simplicity of two-dimensional gravity.

Nevertheless, it is not a very satisfactory situation that observables like the HartleHawking wave-function which have a direct generalization to higher dimensional quantum gravity cannot be calculated using continuum field theory in a straight-forward way. One of the purposes of the study of two-dimensional quantum gravity is to gain experience in the calculation of reparametrization invariant observables in a continuum framework. Although generalizations exist of the formalism of dynamical triangulations to higher dimensions [13, 14, 15] it is presently not clear that the discretized approach is a viable route to four-dimensional quantum gravity, and it is anyway highly desirable to be able to calculate entirely in a continuum framework the reparameterization invariant observables known from the discretized approach. As a first step we calculate in the present paper the lowest orders of quantum corrections to macroscopic loop functions in Liouville theory.

\section{The semi-classical limit}

The starting point of the semi-classical expansion is the partition function for two-dimensional gravity coupled to a conformal field theory with central charge $c<1$. We will be interested in the particular situation where the universe has one boundary, or, alternatively, in the partition function for an open non-critical string theory. f After integration over the matter fields we obtain the following partition function

$$
Z=\int[\mathcal{D} \phi]_{\hat{g}} \mathrm{e}^{-S_{L}(\phi, \hat{g})},
$$

\footnotetext{
${ }^{4}$ A semiclasical analysis of random surfaces with topology of a sphere was performed in [16]. Semiclassical limit of open non-critical string theory was also studied in [17].
} 
where $S_{L}(\phi, \hat{g})$ is the Liouville action on a surface $M$ with boundary $\partial M[18$ :

$$
\begin{aligned}
S_{L}(\phi, \hat{g})=\frac{1}{8 \pi} & {\left[\int_{M} \mathrm{~d}^{2} \xi \sqrt{\hat{g}} \hat{g}^{a b} \partial_{a} \phi \partial_{b} \phi+Q \int_{M} \mathrm{~d}^{2} \xi \sqrt{\hat{g}} \hat{R} \phi+Q \oint_{\partial M} \mathrm{~d} \hat{s} \hat{k} \phi\right] } \\
+ & {\left[\nu \oint_{\partial M} \mathrm{~d} s k-\nu \oint_{\partial M} \mathrm{~d} \hat{s} \hat{k}+t_{0} \int_{M} \mathrm{~d}^{2} \xi \sqrt{\hat{g}} \mathrm{e}^{\alpha \phi}+\zeta_{0} \oint_{\partial M} \mathrm{~d} \hat{s} \mathrm{e}^{\frac{1}{2} \alpha \phi}\right] . }
\end{aligned}
$$

In this formula we have used the standard notation

$$
g_{a b}=\mathrm{e}^{\frac{2}{Q} \phi} \hat{g}_{a b}, \quad Q=\sqrt{\frac{25-c}{3}}, \quad \alpha=\frac{1}{2}\left(Q-\sqrt{Q^{2}-8}\right),
$$

where $\phi$ is the Liouville field, $\hat{g}_{a b}$ is a fixed reference metric with scalar curvature $\hat{R}$ and extrinsic curvature $\hat{k}$ at the boundary. Finally, $t_{0}$ and $\zeta_{0}$ denote the bulk and boundary cosmological coupling constants, respectively. The cosmological terms are needed as short distance counter terms due to short distance singularities. The extrinsic curvature term is not associated with divergences, but is in general needed since the Gauss-Bonnet theorem,

$$
\int_{M} \mathrm{~d}^{2} \xi \sqrt{g} R+\oint_{\partial M} \mathrm{~d} s k=4 \pi \chi(M)
$$

fixes only the sum of the intrinsic and extrinsic curvature terms. From the definition $k n^{b}=-2 t^{a} \nabla_{a} t^{b}$, where $t^{a}$ and $n^{a}$ are the tangent and outwards unit normal to the boundary and $\nabla_{a}$ the covariant derivative, we obtain

$$
k=\mathrm{e}^{-\frac{\phi}{Q}}\left(\hat{k}+\frac{2}{Q} \partial_{\hat{n}} \phi\right)
$$

where $\partial_{\hat{n}}=\hat{n}^{a} \partial_{a}$. (Here the definition of $k$ is different from that in the first Ref of [18] by a factor -2 .)

Eq. (2.5) is analogous to the relation between $\hat{R}$ and $R$ given by

$$
R=\mathrm{e}^{-\frac{2}{Q} \phi}\left(\hat{R}-\frac{2}{Q} \hat{\triangle} \phi\right)
$$

In (2.6) we have used the following notation

$$
\hat{\triangle}=\frac{1}{\sqrt{\hat{g}}} \partial_{a} \sqrt{\hat{g}} \hat{g}^{a b} \partial_{b}=\hat{g}^{a b} \hat{\nabla}_{a} \hat{\nabla}_{b}
$$

where $\hat{\nabla}_{a}$ is the covariant derivative with respect to the metric $\hat{g}_{a b}$. Eq. (2.5) implies

$$
\oint_{\partial M} \mathrm{~d} s k=\frac{2}{Q} \oint_{\partial M} \mathrm{~d} \hat{s} \partial_{\hat{n}} \phi+\oint_{\partial M} \mathrm{~d} \hat{s} \hat{k}
$$

The last term is just a constant and will play no role.

In the following we will for simplicity assume that $M$ has the topology of the disk, since we expect all the additional complications associated with the macroscopic boundary to be present independent of the topology of the "bulk". Given this topology of $M$ we can choose the reference metric as

$$
\hat{g}_{a b}=\delta_{a b}, \quad \text { i.e. } \quad \hat{R}=0, \quad \hat{k}=2 .
$$

The partition function (2.1) with the action (2.2) is a consequence of the conformal anomaly, and the $D D K$ ansatz which allows us to treat the functional measure for $\phi$ as a 
Gaussian measure for an ordinary scalar field. Since $\hat{g}$ is fiducial, the requirement that $Z$ should be independent of $\hat{g}$ implies the relation between $\alpha$ and $Q$ given in (2.3).

The starting point for a semi-classical expansion is the solution $\phi_{c l}$ of the classical equations of motion. One then makes a formal expansion in large $Q$, i.e. we write

$$
\phi=\phi_{c l}+\tilde{\phi}
$$

where $\phi_{c l}$ is of order $Q$ and $\tilde{\phi}$ is of order 1 . The classical equation of motion corresponding to the action (2.2) is

$$
R(g)=-4 \pi t_{0}
$$

where $R(g)$ is the curvature given by (2.6).

For a positive cosmological constant $t_{0}$ (which we assume) there is no solution if the topology is that of the disk. However, if we impose the restriction of a constant area it is possible to perform a semi-classical expansion. Thus we write the partition function as

$$
Z\left(t_{0}, \zeta_{0}\right)=\int_{0}^{\infty} \mathrm{d} A \mathrm{e}^{-t_{0} A} \int_{0}^{\infty} \mathrm{d} \ell \mathrm{e}^{-\zeta_{0} \ell} \Gamma(A, \ell)
$$

In formula (2.12) $\ell \Gamma(A, \ell)$ denotes the disk amplitude $\omega(A, \ell)$ as usually calculated by matrix models for fixed area and fixed length of the boundary. The path integral which defines $\Gamma(A, \ell)$ includes the integration over all metrics on the disk with fixed area $A$ and fixed boundary length $\ell$.

If we implement the area and perimeter constraints

$$
\begin{aligned}
& \int_{M} \mathrm{~d}^{2} \xi \sqrt{\hat{g}} \mathrm{e}^{\alpha \phi}=A, \\
& \oint_{\partial M} \mathrm{~d} \hat{s} \mathrm{e}^{\frac{1}{2} \alpha \phi}=\ell
\end{aligned}
$$

via Lagrange multipliers $\mu_{1}$ and $\mu_{2}$ the action (2.2) is changed to a new action $S_{L}\left(\phi, \mu_{2}, \mu_{1}\right)$ by the replacement

$$
\begin{gathered}
t_{0} \int_{M} \mathrm{~d}^{2} \xi \sqrt{\hat{g}} \mathrm{e}^{\alpha \phi} \rightarrow \mu_{2}\left(\int_{M} \mathrm{~d}^{2} \xi \sqrt{\hat{g}} \mathrm{e}^{\alpha \phi}-A\right) \\
\zeta_{0} \oint_{\partial M} \mathrm{~d} \hat{s} \mathrm{e}^{\frac{1}{2} \alpha \phi} \rightarrow \mu_{1}\left(\oint_{\partial M} \mathrm{~d} \hat{s} \mathrm{e}^{\frac{1}{2} \alpha \phi}-\ell\right) .
\end{gathered}
$$

The starting point of a semi-classical expansion is a stationary point of the action. Under a variation $\phi \rightarrow \phi+\delta \phi$ the action changes as follows

$$
\begin{aligned}
\delta S_{L}\left(\phi, \mu_{2}, \mu_{1}\right)= & \frac{1}{4 \pi} \int_{M} \mathrm{~d}^{2} \xi \partial_{a} \phi \partial_{a} \delta \phi+\mu_{2} \alpha \int_{M} \mathrm{~d}^{2} \xi \mathrm{e}^{\alpha \phi} \delta \phi \\
& +\frac{Q}{4 \pi} \oint_{\partial M} \mathrm{~d} \hat{s} \delta \phi+\frac{1}{2} \mu_{1} \alpha \oint_{\partial M} \mathrm{~d} \hat{s} \mathrm{e}^{\frac{1}{2} \alpha \phi} \delta \phi+\nu \alpha \oint_{\partial M} \mathrm{~d} \hat{s} \partial_{\hat{n}} \delta \phi \\
= & \frac{1}{4 \pi} \int_{M} \mathrm{~d}^{2} \xi \delta \phi\left(-\partial_{a}^{2} \phi+4 \pi \mu_{2} \alpha \mathrm{e}^{\alpha \phi}\right) \\
& +\frac{1}{4 \pi} \oint_{\partial M} \mathrm{~d} \hat{s} \delta \phi\left(\partial_{\hat{n}} \phi+Q+2 \pi \mu_{1} \alpha \mathrm{e}^{\frac{1}{2} \alpha \phi}\right) \\
& +\nu \alpha \oint_{\partial M} \mathrm{~d} \hat{s} \partial_{\hat{n}} \delta \phi .
\end{aligned}
$$

\footnotetext{
${ }^{5}$ The factor $\ell$ is present since the matrix models disk amplitude is usually calculated for a boundary with a marked point.
} 
Let us first consider the case where $\nu=0 . \delta S_{L}\left(\phi, \mu_{2}, \mu_{1}\right)=0$ requires that $\phi$ inside $M$ is a solution to the Liouville equation:

$$
\partial_{a}^{2} \phi=4 \pi \mu_{2} \alpha \mathrm{e}^{\alpha \phi}
$$

On the boundary $\partial M$ we cannot impose the Dirichlet condition on $\phi$, since $\phi$ (being related to det $g_{a b}$ ) is not a scalar field. The Dirichlet boundary condition imposed on $\phi$ breaks the invariance under reparametrizations of the boundary, and this invariance is necessary for being able to choose the conformal gauge. In order to allow $\delta \phi \neq 0$ at the boundary, we have to impose

$$
\partial_{\hat{n}} \phi+Q+2 \pi \mu_{1} \alpha \mathrm{e}^{\frac{1}{2} \alpha \phi}=0 \quad \text { on } \quad \partial M .
$$

This mixed type condition (2.19) keeps the reparametrization symmetry of the boundary intact. Finally the variations with respect to the Lagrange multipliers enforce the constraints (2.13) and (2.14).

A solution up to coordinate transformation is given by

$$
\phi_{c l}(\xi)=\frac{1}{\alpha} \ln \frac{y}{\left(1-x \xi^{2}\right)^{2}},
$$

where $\left(\xi_{1}, \xi_{2}\right)$ are Cartesian coordinates of the unit disk and where we have used the following parameterization [9]:

$$
x=1-\frac{4 \pi A}{\ell^{2}}<1 \quad y=4 \frac{A^{2}}{\ell^{2}}=\frac{A}{\pi}(1-x) .
$$

The area $A$ and the perimeter $\ell$ are related to the values of $\mu_{1}$ and $\mu_{2}$ by

$$
\mu_{2}(x, y)=\frac{2}{\pi \alpha^{2}} \frac{x}{y}, \quad \mu_{1}(x, y)=\frac{1}{2 \pi \alpha \sqrt{y}}(-Q(1+x)+2 \alpha x) .
$$

The action of this classical solution (with general $\nu$ ) can easily be calculated and we obtain

$$
S_{L}\left(\phi_{c l}\right)=\left(\frac{Q}{\alpha}+\frac{16 \pi \nu}{Q \alpha}-1\right)\left(\frac{1}{1-x}-1\right)+\frac{Q}{2 \alpha} \log y-\log (1-x),
$$

and in terms of the original variables $\ell$ and $A$ we can write

$$
\mathrm{e}^{-S_{L}(\phi)}=\text { const. } A^{-\frac{Q}{\alpha}+1} \ell^{\frac{Q}{\alpha}-2} \mathrm{e}^{-\frac{1}{4 \pi}\left(\frac{Q}{\alpha}+\frac{16 \pi \nu}{Q \alpha}-1\right) \frac{\ell^{2}}{A}} .
$$

The functional integral has to be performed under the constraint (2.13), (2.14). Thus it is natural to consider a more general boundary condition of the form

$$
\partial_{\hat{n}} \phi+\frac{1}{2} \hat{k} Q+2 \pi \alpha \mu_{1}(x, y) \mathrm{e}^{\frac{1}{2} \alpha \phi}=\frac{1}{\ell} B(x)\left(\oint_{\partial M} d \hat{s} e^{\frac{1}{2} \alpha \phi}-\ell\right) \quad \text { on } \quad \partial M
$$

where $\hat{k}=2$ for our choice (2.9) of background metric. This boundary condition is of a mixed type and also involves an integral of $e^{\frac{1}{2} \alpha \phi}$ along the boundary. We note that (2.25) is invariant under the transformation

$$
\hat{g}_{a b} \rightarrow \mathrm{e}^{\sigma} \hat{g}_{a b}, \quad \phi \rightarrow \phi-\frac{Q}{2} \sigma,
$$

provided $\alpha$ is given by (2.3), because $\mathrm{e}^{\frac{1}{2} \alpha \phi}$ has, like the bulk cosmological term, an extra $\hat{g}$ dependence due to the reparametrization-invariant regularization and it is transformed in 
the same way as a normal ordered operator in quantum Liouville theory. The right-hand side of (2.25) vanishes due to 2.14). Nonetheless this generalization will yield non-trivial results because a different choice of $B(x)$ defines a different integration measure $\mathcal{D} \phi$.

We note that (2.25) and (2.8) determine an integral of $k$ completely.

$$
\oint_{\partial M} d s k=-4 \pi\left(1-\frac{2 \alpha}{Q}\right)+\frac{2 \ell^{2}}{A}\left(1-\frac{\alpha}{Q}\right)
$$

The Gauss-Bonnet theorem (2.4) then gives

$$
\int_{M} d^{2} \xi \sqrt{g} R=\left(1-\frac{\alpha}{Q}\right)\left(8 \pi-\frac{2 \ell^{2}}{A}\right)
$$

which does not approach the spherical value $8 \pi$ in the limit $\ell \rightarrow 0$. This indicates that the boundary and bulk cosmological terms may be renormalized by finite factors $1-(\alpha / Q)$ and $(1-(\alpha / Q))^{2}$, respectively, in addition to the infinite, $\hat{g}$-dependent factors. This point, however, will not affect the results in this paper.

For the boundary condition (2.25) it follows that $S_{L}$ in (2.17) is stationary for any variation $\delta \phi$ with respects the constraints (2.13) and (2.14), even if $\nu \neq 0$, the reason being that to lowest order in $\delta \phi$ we have

$$
\partial_{\hat{n}} \delta \phi=-\pi \alpha^{2} \mu_{1}(x, y) e^{\frac{1}{2} \alpha \phi} \delta \phi
$$

and consequently the last two terms in (2.17) will vanish due to the constraint (2.14) which implies that

$$
\oint_{\partial M} d \hat{s} e^{\frac{1}{2} \alpha \phi} \delta \phi=0
$$

to first order in $\delta \phi$.

Now the quantum fluctuation $\tilde{\phi}$ will satisfy the boundary condition

$$
\partial_{\hat{n}} \tilde{\phi}=\left(\frac{2(1+x)}{\alpha(1-x)}+\alpha\right)\left(e^{\frac{1}{2} \alpha \tilde{\phi}}-1\right)+B(x) \oint_{\partial M} \mathrm{~d} \hat{s}\left(e^{\frac{1}{2} \alpha \tilde{\phi}}-1\right) \quad \text { at } \quad \partial M,
$$

and to lowest order of expansion in $1 / Q$ (2.31) reads

$$
\partial_{\hat{n}} \tilde{\phi}=\frac{1+x}{1-x} \tilde{\phi}+\frac{1}{2} \alpha B(x) \oint_{\partial M} d \hat{s} \tilde{\phi} .
$$

By performing a decomposition of $\tilde{\phi}$ into modes $\tilde{\phi}_{0}=\oint(d \hat{s} / 2 \pi) \tilde{\phi}$ which are constant at the boundary and $\tilde{\phi}_{1}$ which satisfy $\oint d \hat{s} \tilde{\phi}_{1}=0$, and by defining a new constant $E(x)$ by

$$
B(x)=-\frac{1}{\pi \alpha}\left(\frac{1+x}{1-x}+\frac{2 E(x)}{(1-x)^{2}}\right)
$$

we finally write the boundary condition as

$$
\begin{gathered}
\partial_{\hat{n}} \tilde{\phi}_{1}=\frac{1+x}{1-x} \tilde{\phi}_{1}, \\
\partial_{\hat{n}} \tilde{\phi}_{0}=\frac{-2 E(x)}{(1-x)^{2}} \tilde{\phi}_{0} .
\end{gathered}
$$


(2.34) and (2.35) are the most general boundary conditions compatible with invariance under reparametrizations of the boundary. In Appendix A we will show that $E(x)$ must have an expansion

$$
E(x)=E_{-1}(1-x)+E_{0}+\frac{E_{1}}{1-x}+\cdots,
$$

If we set $B(x)=0$ in (2.25), we will have $E(x)=-\frac{1}{2}\left(1-x^{2}\right)$ and this will not satisfy the above criterion. In later sections we will discuss how to determine $E(x)$.

\section{The one-loop correction}

\subsection{The eigenvalues of the quadratic action}

According to the discussion in the last section we have to perform the following functional integral

$$
Z_{1-\text { loop }}(A, \ell)=\int \frac{[\mathcal{D} \tilde{\phi}]_{\hat{g}}}{V o l_{\hat{g}} P S L(2, \boldsymbol{R})} \mathrm{e}^{-S_{L}^{(2)}(\tilde{\phi})} \delta\left(\int_{M} \mathrm{~d}^{2} \xi \mathrm{e}^{\alpha \phi_{c l}} \tilde{\phi}\right) \delta\left(\int \mathrm{d} \hat{s} \mathrm{e}^{\frac{1}{2} \alpha \phi_{c l}} \tilde{\phi}\right),
$$

where the quadratic action is given by

$$
S^{(2)}(\tilde{\phi})=\frac{1}{8 \pi} \int_{M} \mathrm{~d}^{2} \xi \tilde{\phi}\left[-\partial_{a}^{2}+\frac{8 x}{\left(1-x \xi^{2}\right)^{2}}\right] \tilde{\phi}
$$

and the boundary conditions are given by (2.34)-(2.35). In the conformal gauge on a disk there is a residual gauge symmetry given by $P S L(2, \boldsymbol{R})$ and the group of isometries is its subgroup $S O(2)$. Therefore there are two zero modes and to avoid over-counting we have to divide the functional measure by the volume of this group.

In order to calculate the eigenvalues of the second order differential operator it is convenient to change to spherical coordinates, since the classical solution for $x<0$ describes a spherical cap. We have

$$
\xi^{1} \pm i \xi^{2}=\frac{1}{\sqrt{-x}} \tan \frac{\theta}{2} \mathrm{e}^{ \pm i \varphi}
$$

where

$$
0 \leq \varphi<2 \pi, \quad 0 \leq \theta \leq \operatorname{Tan}^{-1} \sqrt{-x} \equiv \theta_{M}
$$

In terms of the spherical variable we have

$$
S^{(2)}(\tilde{\phi})=\frac{1}{8 \pi} \int_{0}^{2 \pi} \mathrm{d} \varphi \int_{0}^{\theta_{M}} \sin \theta \mathrm{d} \theta \tilde{\phi}[-\hat{\triangle}-2] \tilde{\phi},
$$

where $\hat{\triangle}$ is the Laplacian on the spherical cap (3.3)-(3.4):

$$
\hat{\triangle} \equiv \frac{1}{\sin \theta} \frac{\partial}{\partial \theta} \sin \theta \frac{\partial}{\partial \theta}+\frac{1}{\sin ^{2} \theta} \frac{\partial^{2}}{\partial \varphi^{2}} .
$$

Thus the functions on the spherical cap which diagonalize the quadratic action (3.5) are the generalized spherical harmonics satisfying

$$
-\hat{\triangle} \tilde{\phi}=\lambda_{N m}\left(\lambda_{N m}+1\right) \tilde{\phi} .
$$

They are defined in terms of the associated Legendre functions:

$$
Y_{N m}(\theta, \varphi)=\mathcal{N}_{N m} e^{i m \varphi} P_{\lambda_{N m}^{m}}^{m}(\cos \theta),
$$


where $N=0,1,2, \cdots, m=-N,-N+1, \cdots, N$ and

$$
P_{\lambda}^{m}(w)=(-1)^{m}\left(1-w^{2}\right)^{\frac{|m|}{2}} \frac{\mathrm{d}^{|m|} P_{\lambda}(w)}{\mathrm{d} w^{|m|}} .
$$

For the chosen boundary conditions the eigenvalues $\lambda_{N m}$ will coincide with the ordinary eigenvalues of the sphere in the limit where $x \rightarrow-\infty$, i.e. the limit where $\theta_{M} \rightarrow \pi$ and the cap becomes a sphere. Consequently, we can label the eigenvalues $\lambda_{N m}$ as done in (3.7). The normalization constants $\mathcal{N}_{N m}$ are determined by

$$
\int_{0}^{\theta_{M}} \sin \theta \mathrm{d} \theta \int_{0}^{2 \pi} \mathrm{d} \varphi Y_{N^{\prime} m^{\prime}}^{*}(\theta, \varphi) Y_{N m}(\theta, \varphi)=8 \pi^{2} \delta_{N^{\prime} N} \delta_{m^{\prime} m}
$$

Finally the boundary conditions (2.34)-(2.35) can be written in terms of the associated Legendre functions as

$$
\left(\lambda_{N m}+2\right)(1+x) P_{\lambda_{N m}}^{m}\left(\cos \theta_{M}\right)=\left(\lambda_{N m}-|m|+1\right) P_{\lambda_{N m}-1}^{m}\left(\cos \theta_{M}\right)
$$

for $m \neq 0$ and, for $m=0$, as

$$
\left[\frac{2 E(x)}{1-x}+\lambda_{N 0}(1+x)\right] P_{\lambda_{N 0}}\left(\cos \theta_{M}\right)=\lambda_{N 0}(1-x) P_{\lambda_{N 0}-1}\left(\cos \theta_{M}\right) .
$$

In Appendix A we outline how to find the eigenvalues and the normalization constants as an expansion in $1 /(1-x)$.

\subsection{The functional integral}

We use the expansion

$$
\tilde{\phi}=\sum_{N=0}^{\infty} \sum_{m=-N}^{N} a_{N m} Y_{N m}(\theta, \varphi), \quad a_{N,-m}=a_{N m}^{*}
$$

in (3.1) and have to express the area and perimeter constraints in terms of the mode expansion (3.13):

$$
\begin{gathered}
\int_{M} \mathrm{~d}^{2} \xi \mathrm{e}^{\alpha \phi_{c l}} \tilde{\phi}=-\frac{\sqrt{2 \pi} y}{4 x} \sum_{N=0}^{\infty} a_{N 0} \mathcal{N}_{N 0} \int_{\cos \theta_{M}}^{1} d w P_{\lambda_{N 0}}(w) \equiv-\frac{\sqrt{2 \pi}}{4} \sum_{N=0}^{\infty} \alpha_{N} a_{N 0} \\
\int \mathrm{d} \hat{s} \mathrm{e}^{\frac{1}{2} \alpha \phi_{c l}} \tilde{\phi}=\frac{2 \pi \sqrt{y}}{1-x} \sum_{N=0}^{\infty} a_{N 0} \mathcal{N}_{N 0} P_{\lambda_{N 0}}\left(\cos \theta_{M}\right) \equiv 2 \pi \sum_{N=0}^{\infty} \beta_{N} a_{N 0} .
\end{gathered}
$$

Using the integration formula for $P_{\lambda}(w)$ and the relation between hyper-geometric functions and associated Legendre polynomials 20] we have the following explicit expressions for the coefficients $\alpha_{N}$ and $\beta_{N}$ :

$$
\begin{aligned}
\alpha_{N} & =\frac{y}{-x} \mathcal{N}_{N 0} \frac{\sin \theta_{M}}{\lambda_{N 0}\left(\lambda_{N 0}+1\right)} P_{\lambda_{N 0}}^{1}\left(\cos \theta_{M}\right) \\
& =\frac{y}{(1-x)^{2}} \mathcal{N}_{N 0} F\left(1-\lambda_{N 0}, 2+\lambda_{N_{0}} ; 2 ; \frac{-x}{1-x}\right) \\
\beta_{N} & =\frac{\sqrt{y}}{1-x} \mathcal{N}_{N 0} P_{\lambda_{N 0}}\left(\cos \theta_{M}\right)=\frac{\sqrt{y}}{1-x} \mathcal{N}_{N 0} F\left(-\lambda_{N 0}, 1+\lambda_{N_{0}} ; 1 ; \frac{-x}{1-x}\right) .
\end{aligned}
$$


A shift of variables from $\tilde{\phi}$ to $\left\{a_{N m}\right\}$ transforms the one-loop integral (3.1) to

$$
\begin{aligned}
Z_{1-\text { loop }}(A, \ell)= & \left(\prod_{N, m} \int \mathrm{d} a_{N m}\right) \exp \left\{-\pi \sum_{N=0}^{\infty} \sum_{m=-N}^{N}\left[\lambda_{N m}\left(\lambda_{N m}+1\right)-2\right]\left|a_{N m}\right|^{2}\right\} \\
& \times \delta\left(\sum_{N} \alpha_{N} a_{N 0}\right) \delta\left(\sum_{N} \beta_{N} a_{N 0}\right) \delta\left(a_{11}\right) \delta\left(a_{1,-1}\right) \times \mathcal{J} \times \Delta_{F P},
\end{aligned}
$$

where the last two $\delta$-functions are caused by the zero modes corresponding to $Y_{1, \pm 1}$ and $\Delta_{F P}$ is the Faddeev-Popov determinant. Because we adopted the normalization (3.10), we have to shift from the functional measure $[\mathcal{D} \tilde{\phi}]_{\hat{g}}$ to $[\mathcal{D} \tilde{\phi}]_{\breve{g}}$, where $\breve{g}_{a b}=-\frac{1}{2 \pi^{2}} \frac{x}{y} e^{\alpha \phi_{c l}} \hat{g}_{a b}$. This induces a Weyl anomaly and is the origin of the Jacobian $\mathcal{J}$. Calculation of $\Delta_{F P}$ and $\mathcal{J}$ is outlined in Appendix B. Except for the $\delta$-functions the integrals can be performed and we obtain

$$
\mathcal{J} \times \Delta_{F P} \times \hat{K} \times \hat{I}
$$

where

$$
\hat{K} \equiv\left[\prod_{N=1}^{\infty} \prod_{m=1}^{N} \frac{1}{\lambda_{N m}\left(\lambda_{N m}+1\right)-2}\right]
$$

and where $\hat{I}$ is the integral over the $a_{N 0}$ 's. The prime in $\Pi^{\prime}$ means that the factor for $N=m=1$ must be excluded from the product. We can implement the two $\delta$-functions via Lagrange multipliers and since the integrations over the $a_{N 0}$ 's and the Lagrange multipliers are Gaussian, we get after a straightforward calculation:

$$
\hat{I}=\sqrt{\prod_{N=0}^{\infty} \frac{1}{\lambda_{N 0}\left(1+\lambda_{N 0}\right)-2}} \times \frac{1}{\sqrt{X Z-Y^{2}}}
$$

where

$$
X=\sum_{N=0}^{\infty} \frac{\alpha_{N}^{2}}{\lambda_{N 0}\left(1+\lambda_{N 0}\right)-2}, \quad Y=\sum_{N=0}^{\infty} \frac{\alpha_{N} \beta_{N}}{\lambda_{N 0}\left(1+\lambda_{N 0}\right)-2}, \quad Z=\sum_{N=0}^{\infty} \frac{\beta_{N}^{2}}{\lambda_{N 0}\left(1+\lambda_{N 0}\right)-2} .
$$

The problem is now reduced to the calculation of the infinite products and sums which appear in (3.19)-(3.22). It is a two-fold problem. We have to calculate the eigenvalues $\lambda_{N m}$ and afterwards we have to use these values in the actual calculation of the infinite sum and products. It seems impossible to calculate the eigenvalues for a general geometry, but two cases can be treated: $x=-1$, which corresponds to $\theta_{M}=\pi / 2$, i.e. to the geometry of a bowl, and the limit $x \rightarrow-\infty$, i.e. the limit where $\ell^{2} / A \rightarrow 0$. In Appendix $\mathrm{A}$ the calculation of the eigenvalues in the limit $x \rightarrow-\infty$ is sketched and it is shown that $E(x)$ must have an expansion (A.3) in order for the eigenvalues to coincide with those of the sphere in the limit $x \rightarrow-\infty$. We note that the expansions for the eigenvalues $\lambda_{N 0}$ contain $\ln (1-x)$. We can also show that the expansions for other $\lambda_{N m}$ generally contain $\ln (1-x)$ in higher orders.

The next problem is the calculation of the infinite products. This is again non-trivial from a technical point of view. Using the results in Appendix A we obtain after a somewhat lengthy calculation the following result:

$$
\begin{aligned}
\hat{I} \propto & \frac{(1-x)^{2}}{y^{3 / 2}} \exp \left\{\frac { 1 } { 1 - x } \left\{-\left(E_{-1}+2\right) \ln (1-x)-1-\frac{1}{2} \sum_{N=2}^{\infty}(2 N+1)\right.\right. \\
& \left.\left.-\left(E_{-1}+2\right) \sum_{N=2}^{\infty} \frac{2 N+1}{(N-1)(N+2)}\right\}+O\left((1-x)^{-2}\right)\right\}
\end{aligned}
$$


and

$$
\hat{K} \propto 1+O\left((1-x)^{-2}\right) .
$$

The infinite sums which appear in (3.23) may be regularized by the zeta-function regularization [19]. However, it turns out that infinities get more severe in the next orders of $1 /(1-x)$. This means that the infinite product and the expansion in $1 /(1-x)$ do not commute.

Therefore we turned to evaluate $\hat{I}$ by exploiting the zeta-function regularization without explicitly computing the eigenvalues. Some details of this calculation is provided in Appendix C. The results are

$$
\begin{gathered}
\sqrt{\prod_{N=0}^{\infty} \frac{1}{\lambda_{N 0}\left(1+\lambda_{N 0}\right)-2}}=\frac{(1-x)^{5 / 4}(-x)^{1 / 8}}{\sqrt{2 \sqrt{\pi}(1+x)\left(E(x)+\frac{2 x(1-x)}{1+x}\right)}} \\
\frac{1}{\sqrt{X Z-Y^{2}}}=\frac{(-x)^{3 / 4}(1-x) \sqrt{3+x^{2}}}{8 \pi y^{3 / 2} \sqrt{\theta_{M}}}\left[\frac{2 x}{1+x} \frac{E(x)+\frac{1}{2}(1-x)(3+x)}{E(x)+\frac{2 x(1-x)}{1+x}}+\ln \frac{1}{1-x}\right]^{-\frac{1}{2}}
\end{gathered}
$$

and the product of the two yields

$$
\begin{gathered}
\hat{I}=\quad \frac{(-x)^{7 / 8}(1-x)^{9 / 4}}{2 y^{3 / 2}} \sqrt{\frac{3+x^{2}}{(4 \pi)^{5 / 2} \theta_{M}}}\left[2 x\left(E(x)+\frac{1}{2}(1-x)(3+x)\right)\right. \\
\left.+(1+x)\left(E(x)+\frac{2 x(1-x)}{1+x}\right) \ln \frac{1}{1-x}\right]^{-\frac{1}{2}}
\end{gathered}
$$

Now all $E(x)$ dependence is contained in $(3.27)$. In this equation we notice a logarithm $\ln (1-x)$ which will cause a factor $\left(\ell^{2} / A\right)^{\left(\ell^{2} / A\right)}$ in $Z_{1 \text {-loop }}$ in the limit $\ell^{2} / A \rightarrow 0$. On the other hand the matrix model result for the partition function for some special background 8, 9]

$$
Z_{\text {matrix }}=\ell^{\frac{Q}{\alpha}-3} A^{-\frac{Q}{\alpha}} e^{- \text {const. } \frac{\ell^{2}}{A}}
$$

does not contain such a factor and to reproduce the matrix model result this logarithm has to be canceled in the product $\hat{I} \times \hat{K}$. This requirement will determine $E(x)$ completely. Let us notice the following feature of $(3.25) \sim(3.27)$. The logarithm in $\hat{I}$ comes solely from (3.26), i.e. the area and perimeter constraints, and not from (3.25), i.e. the product of the eigenvalues. This result is somewhat surprising because the expansions of $\lambda_{N 0}$ about the limit $x \rightarrow-\infty$ do contain $\ln (1-x)$ 's as shown in Appendix A. This observation leads us to conjecture that $\hat{K}$ does not contain $\ln (1-x)$, either. Actually, when we compute

$$
\hat{K}_{m}=\prod_{N=\max \{2, m\}}^{\infty} \frac{1}{\lambda_{N m}\left(\lambda_{N m}+1\right)-2}
$$

for each $m \geq 1$ by the zeta-function regularization explained in Appendix C, we obtain

$$
\hat{K}_{1}=\frac{9(1-x)^{3}}{\pi(-x)^{5 / 2}(3-x)^{2}}, \quad \hat{K}_{m}=\frac{m^{2}\{(m-2) !\}^{2}}{\pi(1-x)(-x)^{m-\frac{1}{2}}} \quad(m \geq 2) .
$$


This is a strong argument in favor of the absence of $\ln (1-x)$ in $\hat{K}=\prod_{m=1}^{\infty} K_{m}$, although it is of course not a rigorous proof. If $\ln (1-x)$ is indeed absent in $\hat{K}$ we will have to eliminate $\ln (1-x)$ in (3.27) by choosing

$$
E(x)=\frac{-2 x(1-x)}{1+x},
$$

and thus the boundary condition has been completely determined by the comparison with the known matrix model results.

\section{Discussion}

In this paper we have investigated the macroscopic wave functions of non-critical string theory within the framework of the semi-classical expansion. We have singled out the most general boundary condition for the Liouville field that does not break reparametrization invariance of the boundary. This condition contains one unknown function $E(x)$ of $x=$ $1-4 \pi\left(A / \ell^{2}\right)$. The requirement that terms with $\ln (1-x)$ have to be absent in the wave function was used to determine $E(x)$. To determine this function we (partially) computed the lowest order quantum correction to the wave function. Then we determined $E(x)$ to be given by (3.31) by calculating the contribution $\hat{I}$ to the wave function from the constant modes on the boundary $\tilde{\phi}_{0}$ and by arguing (and partly conjecture) that the remaining part, $\hat{K}$, in the wave function does not contain $\ln (1-x)$.

The boundary condition (3.31) has interesting property: (3.25) becomes infinite when we substitute (3.31). This is due to a new zero mode $Y_{1,0}$ corresponding to the dilatation $\xi \rightarrow e^{\beta} \xi$ which accidentally appears in the quadratic action for this boundary condition. Even in this case (3.27) remain finite because (3.26) vanishes. This is quite reasonable because our system does not have dilatation symmetry. However, it is desirable to be able to derive the result (3.31) from first principles, rather than by comparison with matrix model calculations. The above superficial dilatation symmetry may be helpful in this connection.

To complete the one-loop calculation we have to evaluate $\hat{K}$. This work is now in progress and the results will be reported elsewhere 25.

\section{Acknowledgements}

Part of this work was done, while one of the authors (R. N.) visited the Niels Bohr Institute. He is grateful to the hospitality of the staffs and the members of the theoretical high energy group there.

The work of R. N. is supported in part by Grant-in-Aid for Scientific Research ((C) $(2)$ No 07640364), ((A)(1) No 08304024) and Grant-in-Aid for International Scientific Research (Joint Research No 07044048) from the Ministry of Education, Science and Culture of Japan.

J.A. acknowledges the support of the Professor Visitante Iberdrola grant and the hospitality at the University of Barcelona, where part of this work was done.

\section{A Harmonic Analysis on a Disk}

In this appendix we discuss some aspects of harmonic analysis on the disk. As described in the text we have to solve the following eigenvalue problem on the spherical cap with 
coordinates $(\theta, \varphi)$ and $\theta \in\left[0, \theta_{M}\right], 0<\theta_{M} \leq \pi, \cos \theta_{M}=\frac{1+x}{1-x}$ :

$$
-\hat{\triangle} \tilde{\phi}=\left(\lambda_{N m}\left(\lambda_{N m}+1\right)-2\right) \tilde{\phi} .
$$

The boundary condition is specified by performing the decomposition into modes $\tilde{\phi}_{0}$ which are constant at the boundary $\partial M$ and modes $\tilde{\phi}_{1}$ which satisfy $\oint d s \tilde{\phi}_{1}=0$. We have from (2.35)

$$
\partial_{\hat{n}} \tilde{\phi}_{1}=\frac{1+x}{1-x} \tilde{\phi}_{1}, \quad \partial_{\hat{n}} \tilde{\phi}_{0}=\frac{-2 E(x)}{(1-x)^{2}} \tilde{\phi}_{0} .
$$

In general we image that $E(x)$ in (2.35) has an expansion

$$
E(x)=E_{-1}(1-x)+E_{0}+\frac{E_{1}}{1-x}+\cdots,
$$

The special choice (3.31),

$$
E(x)=-\frac{2 x(1-x)}{1+x}=-2(1-x)-2 \sum_{n=0}^{\infty} \frac{2^{n}}{(1-x)^{n}} .
$$

corresponds to the following equation for $\partial_{\hat{n}} \tilde{\phi}_{0}$ in $($ A.2. $)$ :

$$
\partial_{\hat{n}} \tilde{\phi}_{0}=\frac{4 x}{1-x^{2}} \tilde{\phi}_{0}
$$

\section{Expansion of eigenvalues}

It seems difficult to solve the eigenvalue problem for arbitrary $x<0$. Rather, we try an expansion in powers of $\ell^{2} / A$, i.e. in inverse powers of $1-x$. In this case we have that the eigenvalues $\lambda_{N m}$ should behave as

$$
\lambda_{N m}=N+\sum_{n=m}^{\infty} \frac{\Delta_{n}^{(N m)}}{(1-x)^{n}},
$$

In addition we expect the normalization constants $\mathcal{N}_{N m}$ of the spherical harmonics (3.8) to have an expansion

$$
\mathcal{N}_{N m}=2 \sqrt{2} \pi \mathcal{N}_{N m}^{(0)}\left(1+\sum_{n=m}^{\infty} \frac{a_{n}^{(N m)}}{(1-x)^{n}}\right)
$$

where $\mathcal{N}_{N m}^{(0)}$ is the normalization of the ordinary spherical harmonics.

In principle the calculation of the corrections $\Delta_{n}^{(N m)}$ and $a_{n}^{(N m)}$ is straightforward. In practice it is delicate since the associated Legendre functions $P_{\lambda_{N m}}^{m}(w)$ are singular at $w=-1$ unless $\lambda_{N m}$ is an integer and $x \rightarrow-\infty$ corresponds precisely to this limit since $\cos \theta_{M} \rightarrow-1$ for $x \rightarrow-\infty$. The singular behavior can be disentangled by using the representation of $P_{\nu}^{m}(w)$ by hypergeometric functions 20] :

$$
P_{\nu}^{m}(w)=(-1)^{m} \frac{\Gamma(\nu+m+1)}{m ! 2^{m} \Gamma(1+\nu-m)}\left(1-w^{2}\right)^{\frac{m}{2}} F\left(m-\nu, m+\nu+1 ; m+1 ; \frac{1-w}{2}\right) .
$$


In terms of the hypergeometric function the boundary conditions read:

$$
\begin{gathered}
(m-1)\left(1-x^{2}\right) F\left(\lambda_{N m}+m+1, m-\lambda_{N m} ; m+1 ; \frac{-x}{1-x}\right) \\
=2 x F^{\prime}\left(\lambda_{N m}+m+1, m-\lambda_{N m} ; m+1 ; \frac{-x}{1-x}\right), \\
E(x) F\left(\lambda_{N 0}+1,-\lambda_{N 0} ; 1 ; \frac{-x}{1-x}\right)=x F^{\prime}\left(\lambda_{N 0}+1,-\lambda_{N 0} ; 1 ; \frac{-x}{1-x}\right),
\end{gathered}
$$

where A.9) is valid for $m>0$. The hypergeometric functions in (A.9) and (A.10) become singular when $z=-x /(1-x) \rightarrow 1$. But it is well known how to extend the hypergeometric series $F(\alpha, \beta ; \gamma ; z)$, defined inside the unit circle $|z|<1$, to that inside the region $|1-z|<1$ by Gauss's transformation formula (see e.g. 9.154 in [20] for some details). Using the same technique one can separate the singularities and the cut starting at $z=1$. After some calculations one obtains for $M=0,1,2, \cdots$

$$
\begin{aligned}
& F(\alpha, \beta ; \alpha+\beta-M ; z)= \\
& \quad \frac{\Gamma(\alpha+\beta-M)}{\Gamma(\alpha) \Gamma(\beta)} \sum_{n=0}^{M-1}(-1)^{n} \frac{(\alpha-M)_{n}(\beta-M)_{n}(M-n-1) !}{n !(1-z)^{M-n}} \\
& \quad-\frac{(-1)^{M}}{M !} \frac{\Gamma(\alpha+\beta-M)}{\Gamma(\alpha-M) \Gamma(\beta-M)}[F(\alpha, \beta ; M+1 ; 1-z) \ln (1-z) \\
& \left.\quad-\sum_{n=0}^{\infty} \frac{(\alpha)_{n}(\beta)_{n}}{(M+1)_{n} n !}(1-z)^{n}\{\psi(n+1)+\psi(n+M+1)-\psi(n+\alpha)-\psi(n+\beta)\}\right] .
\end{aligned}
$$

Here $\psi(z)=\frac{d}{d z} \ln \Gamma(z)$ is the di-gamma function (psi function). In this way we can explicitly control the singularities at $z=1$.

By inserting (A.11) in the boundary equations (A.9) and (A.10) we can show that $\lambda_{N m}$ approaches the spherical value $N$, only if $E(x)$ has an expansion (A.3) and we obtain after a tedious calculation:

$$
\begin{aligned}
\Delta_{1}^{(N 0)=} & E_{-1}+N(N+1) \\
\Delta_{2}^{(N 0)=} & \left(\Delta_{1}^{(N 0)}\right)^{2}[\ln (1-x)+2(\psi(1+N)-\psi(1))] \\
& +\Delta_{1}^{(N 0)}\left(1-2 N^{2}\right)+E_{0}+\frac{1}{2}[N(N+1)]^{2}, \\
\Delta_{2}^{(N 1)}= & \frac{1}{2}(N+2)(N+1) N(N-1), \\
\Delta_{2}^{(N 2)=} & -\frac{1}{6}(N+2)(N+1) N(N-1),
\end{aligned}
$$

while all other $\Delta_{1,2}^{(N m)}=0$.

\section{The normalization of the spherical harmonics}

The normalization is fixed by the convention (3.10), i.e.

$$
\left(\mathcal{N}_{N m}\right)^{-2}=\frac{1}{4 \pi} \int_{\frac{1+x}{1-x}}^{1} \mathrm{~d} w\left(P_{\lambda_{N m}}^{m}(w)\right)^{2} \equiv U_{\lambda_{N m}}^{m} .
$$


Using standard recursion relations between $P_{\lambda}^{m}, P_{\lambda}^{m-1}$ and $\left(P_{\lambda}^{m}\right)^{\prime}$ one derives the following recursion relation between $U_{\lambda}^{m}$ :

$$
\begin{aligned}
U_{\lambda}^{m}= & (\lambda-m+1)(\lambda+m) U_{\lambda}^{m-1} \\
+ & \frac{1}{4 \pi}\left(\frac{-4 x}{(1-x)^{2}}\right)^{m} P_{\lambda}^{m-1}\left(\frac{1+x}{1-x}\right) P_{\lambda}^{m}\left(\frac{1+x}{1-x}\right) .
\end{aligned}
$$

This leave us with the task of calculation of $U_{\lambda}^{0}$.

As for the eigenvalues $\lambda_{N m}$, it seems difficult to determine $\mathcal{N}_{N m}$ except as an expansion in inverse powers of $1-x$. Following (A.18) we write

$$
\mathcal{N}_{N m}=\sqrt{2 \pi \frac{(2 N+1)(N-m) !}{(N+m) !}}\left(1+\sum_{n=m}^{\infty} \frac{a_{n}^{(N m)}}{(1-x)^{n}}\right),
$$

and want to determine the first coefficients $a_{n}^{(N m)}$. The basic equation for $U_{\lambda}^{0}$ becomes

$$
\begin{aligned}
4 \pi U_{\lambda}^{0} & =\int_{\frac{1+x}{1-x}}^{1} \mathrm{~d} w\left(P_{\lambda}(w)\right)^{2}=\int_{-1}^{1} \mathrm{~d} w\left(P_{\lambda}(w)\right)^{2}-\int_{-1}^{\frac{1+x}{1-x}} \mathrm{~d} w\left(P_{\lambda}(w)\right)^{2} \\
& =\frac{\pi^{2}-2 \sin ^{2} \pi \lambda \psi^{\prime}(\lambda+1)}{\pi^{2}\left(\lambda+\frac{1}{2}\right)}-\int_{-1}^{\frac{1+x}{1-x}} d w\left[F\left(-\lambda, \lambda+1 ; 1 ; \frac{1-w}{2}\right)\right]^{2}
\end{aligned}
$$

We have expressed the associated Legendre function $P_{\lambda}(w)$ in terms of a hypergeometric function $F$. The singularities at $w=-1$ in the integral (A.19) can now be handled by the transformation formula (A.11) and the integral calculated by expanding the resulting expression in powers of $1+w$ near $w=-1$. In this way one obtain, using the expressions for the eigenvalues $\lambda_{N 0}$, already known from (A.12) and (A.13), the corrections $a_{n}^{(N 0)}$ to $\mathcal{N}_{N 0}$. We can then apply the recursion formula (A.17) to obtain the corrections $a_{n}^{(N m)}$. Again this involves the singularities of $P_{\lambda_{N m}}^{m}(w)$ at $w=-1$, and they too have to be dealt with using the representation of $P_{\lambda_{N m}}^{m}(w)$ in terms of hypergeometric functions. Let us summarize the results of the quite lengthy calculation as follows:

$$
\begin{aligned}
a_{1}^{(N 0)=} & \frac{E_{-1}+N(N+1)}{2 N+1}+\frac{2 N+1}{2}, \\
a_{2}^{(N 0)=} & -\frac{1}{8}\left(4 N^{2}-2 N-3\right)(2 N+1)-(2 N+1) \Delta_{1}^{(N 0)} \ln (1-x) \\
& -\Delta_{1}^{(N 0)}\left\{2(2 N+1)(\psi(1)-\psi(N+1))+2 N-\frac{1}{2}\right\} \\
& +\left(\Delta_{1}^{(N 0)}\right)^{2}\left(\psi^{\prime}(N+1)-\frac{1}{2(2 N+1)^{2}}\right)+\frac{\Delta_{2}^{(N 0)}}{2 N+1}, \\
a_{2}^{(N 1)=} & \frac{\Delta_{2}^{(N 1)}}{2 N+1}, \\
a_{2}^{(N 2)=} & \frac{\Delta_{2}^{(N 2)}}{2 N+1},
\end{aligned}
$$

while all other $a_{1,2}^{(N m)}=0$. 


\section{B Jacobian $\mathcal{J}$ and Faddeev-Popov Determinant $\Delta_{F P}$}

\section{Jacobian $\mathcal{J}$}

The functional measure $[\mathcal{D} \tilde{\phi}]_{\hat{g}}$ is defined with respect to the norm

$$
\|\delta \tilde{\phi}\|_{\hat{g}}^{2}=\int_{M} d^{2} \xi \sqrt{\hat{g}}(\delta \tilde{\phi})^{2},
$$

while $[\mathcal{D} \tilde{\phi}]_{\breve{g}}$ with respect to

$$
\|\delta \tilde{\phi}\|_{\breve{g}}^{2}=-\frac{1}{2 \pi^{2}} \frac{x}{y} \int_{M} d^{2} \xi e^{\alpha \phi_{c l}} \sqrt{\hat{g}}(\delta \tilde{\phi})^{2} .
$$

The Jacobian $\mathcal{J}$ defined by

$$
[\mathcal{D} \tilde{\phi}]_{\hat{g}}=\mathcal{J}[\mathcal{D} \tilde{\phi}]_{\breve{g}}
$$

is given by Weyl anomaly

$$
\mathcal{J}=\exp \left[-\left.\frac{1}{3 Q^{2}} S_{L}\left(\frac{Q}{2}\left(\alpha \phi_{c l}+\ln \left(-\frac{1}{2 \pi^{2}} \frac{x}{y}\right)\right) ; \hat{g}\right)\right|_{t_{0}=\xi_{0}=0}\right] .
$$

By a straightforward calculation we have

$$
\mathcal{J}=\text { const. }(-x)^{-\frac{1}{12}} e^{-\left(\frac{1}{6}+\frac{8 \pi \nu}{3 Q^{2}}\right) \frac{1}{1-x}} .
$$

\section{Faddeev-Popov determinant $\Delta_{F P}$}

The residual symmetry in the conformal gauge is given by

$$
\delta z=-v+v^{*} z^{2} .
$$

Here $v$ is an infinitesimal complex parameter. Under this transformation the variation $\delta \phi$ is given by

$$
\delta \phi=\delta z \partial_{z} \phi+\frac{Q}{2} \partial_{z} \delta z+\text { c.c. }
$$

and that of $a_{1, \pm 1}$ by

$$
\delta a_{1,1}=\left(\delta a_{1,-1}\right)^{*}=\frac{1}{8 \pi^{2}} \int_{0}^{\theta_{M}} \sin \theta d \theta \int_{0}^{2 \pi} d \varphi \delta \phi Y_{1,1}^{*}(\theta, \varphi) .
$$

To leading order in $1 / Q$ this gives

$$
\delta a_{1,1}=\frac{v^{*} Q}{2 \pi} \mathcal{N}_{1,1} \frac{x^{2} \sqrt{-x}}{(1-x)^{2}}
$$

and by using

$$
\mathcal{N}_{1,1}=\frac{\sqrt{3 \pi}(1-x)^{3 / 2}}{-x \sqrt{3-x}}
$$

we obtain as the determinant $\partial\left(a_{1,1}, a_{1,-1}\right) / \partial(v, v *)$

$$
\Delta_{F P}=\text { const. }\left(\mathcal{N}_{1,1}\right)^{2} \frac{-x^{3}}{(1-x)^{4}}=\text { const. } \frac{-x}{(1-x)(3-x)} .
$$




\section{Zeta-Function Regularization}

\section{Calculation of $\hat{I}$}

Zeta-function regularization 19 is known as a powerful method for computing functional determinants. Here we will apply the technique in [21] to the calculation of $\hat{I}$ via zetafunction regularization.

We first define a function

$$
\zeta_{0}(s)=\sum_{N=0}^{\infty}\left(\lambda_{N 0}-1\right)^{-s}\left(\lambda_{N 0}+2\right)^{-s} .
$$

Analysis of (3.12) shows that $\lambda_{N 0}$ increases linearly for large $N$ and the series (C.1) is defined only for Re $s>\frac{1}{2}$. We then analytically continue $\zeta_{0}(s)$ onto the whole complex $s$ plane and afterwards compute the infinite product by

$$
\prod_{N=0}^{\infty} \frac{1}{\sqrt{\lambda_{N 0}\left(1+\lambda_{N 0}\right)-2}}=\exp \left[\frac{1}{2} \zeta_{0}^{\prime}(0)\right] \text {. }
$$

As a first step we will rewrite $\zeta_{0}(s)$ as a contour integral.

$$
\zeta_{0}(s)=\int_{\gamma_{1}} \frac{d \lambda}{2 \pi i}[(\lambda-1)(\lambda+2)]^{-s} \frac{\partial}{\partial \lambda} \ln G_{0}(\lambda ; x)
$$

Here $G_{0}$ is a function defined by

$$
G_{0}(\lambda ; x)=\left[\frac{2 E(x)}{1-x}+\lambda(1+x)\right] P_{\lambda}\left(\frac{1+x}{1-x}\right)-\lambda(1-x) P_{\lambda-1}\left(\frac{1+x}{1-x}\right)
$$

and the complex $\lambda$ plane is cut along the two cuts $(1,1+i \infty),(-2,-2-i \infty)$. The contour $\gamma_{1}$ encircles the poles $\lambda=\lambda_{N 0} \quad(N=0,1,2, \cdots)$ counterclockwise. (See fig.1) show

By using the identity $P_{\lambda}(w)=P_{-\lambda-1}(w)$ and the recursion relation for $P_{\lambda}(w)$ we can

$$
G_{0}(-\lambda-1 ; x)=G_{0}(\lambda ; x)
$$

and thus the integrand of C.3 is antisymmetric under a transformation $\lambda \rightarrow-\lambda-1$. Furthermore in the integrand of (C.3) there is no more singularity than the poles at $\lambda=\lambda_{N 0},-\lambda_{N 0}-1 \quad(N=0,1,2, \cdots)$ and the two cuts. Therefore we can further rewrite (C.3) as

$$
\zeta_{0}(s)=-\int_{\gamma 2} \frac{d \lambda}{2 \pi i}[(\lambda-1)(\lambda+2)]^{-s} \frac{\partial}{\partial \lambda} \ln G_{0}(\lambda ; x),
$$

where the new contour $\gamma_{2}$ encircles the cut $(1,1+i \infty)$ counterclockwise. As long as $\frac{1}{2}<$ Re $s<1$ this integral reduces to that of discontinuity along the cut

$$
\zeta_{0}(s)=\frac{1}{\pi} e^{\frac{\pi}{2} i s} \sin \pi s \quad \int_{0}^{\infty} d t t^{-s}(3+i t)^{-s} \frac{\partial}{\partial t} \ln G_{0}(1+i t ; x) .
$$

In the second step we analytically continue $\zeta_{0}(s)$ to the region $\operatorname{Re} s<\frac{1}{2}$ by using an asymptotic expansion of $P_{\nu}(\cos \theta)$ for $\operatorname{Im} \nu \rightarrow+\infty$ 22],

$$
P_{\nu}(\cos \theta)=\frac{1}{\sqrt{2 \pi \sin \theta}} \frac{\Gamma(\nu+1)}{\Gamma(\nu+3 / 2)} e^{-i\left(\nu \theta+\frac{\theta}{2}-\frac{\pi}{4}\right)}\left(1+O\left(\frac{1}{\nu}\right)\right)+O\left(e^{2 i \nu \theta}\right),
$$


from which we obtain

$$
G_{0}(\lambda ; x) \sim(x-1) \sqrt{\frac{\lambda}{2 \pi} \sin \theta_{M}} e^{-i\left(\lambda \theta_{M}+\frac{1}{2} \theta_{M}-\frac{3 \pi}{4}\right)} \quad \lambda \rightarrow 1+i \infty .
$$

We decompose $\zeta_{0}(s)$ into

$$
\zeta_{0}^{(1)}(s)=\frac{1}{\pi} e^{\frac{i}{2} \pi s} \sin \pi s \quad \int_{0}^{\infty} d t t^{-s}(3+i t)^{-s} \frac{\partial}{\partial t}\left[\ln G_{0}(1+i t ; x)-H_{0}(1+i t ; x)\right]
$$

and

$$
\zeta_{0}^{(2)}(s)=\frac{1}{\pi} e^{\frac{i}{2} \pi s} \sin \pi s \quad \int_{0}^{\infty} d t t^{-s}(3+i t)^{-s} \frac{\partial}{\partial t} H_{0}(1+i t ; x)
$$

where

$$
H_{0}(\lambda ; x)=\ln \left[(x-1) \sqrt{\frac{\sin \theta_{M}}{2 \pi}}\right]+\frac{1}{2} \ln \lambda-i\left(\lambda \theta_{M}+\frac{1}{2} \theta_{M}-\frac{3 \pi}{4}\right) .
$$

Now (C.10) is well defined for $-\frac{1}{2}<\operatorname{Re} s<1$ and we obtain

$$
\begin{aligned}
\zeta_{0}^{(1) \prime}(0) & =\int_{0}^{\infty} d t \frac{\partial}{\partial t}\left[\ln G_{0}(1+i t ; x)-H_{0}(1+i t ; x)\right] \\
& =-\ln G_{0}(1 ; x)+H_{0}(1 ; x)
\end{aligned}
$$

to which we substitute from (C.4)

$$
\ln G_{0}(1 ; x)=\ln \left[\frac{2(1+x)}{(1-x)^{2}}\left\{E(x)+\frac{2 x(1-x)}{1+x}\right\}\right] .
$$

To compute (C.11) we need an integral

$$
\mathcal{I}_{n}(s)=\frac{1}{\pi} e^{\frac{i}{2} \pi s} \sin \pi s \quad \int_{0}^{\infty} d t t^{-s}(3+i t)^{-s}(1+i t)^{-n} .
$$

This is valid only for $\frac{1-n}{2}<\operatorname{Re} s<1$ but can be analytically continued using a formula[23]

$$
\int_{0}^{\infty} d t t^{\alpha-1}(t+a)^{-\beta}(t+b)^{-\gamma}=b^{-\gamma} a^{\alpha-\beta} B(\alpha, \beta+\gamma-\alpha) F\left(\alpha, \gamma ; \beta+\gamma ; 1-\frac{a}{b}\right)
$$

$(0<\operatorname{Re} \alpha<\operatorname{Re}(\beta+\gamma),|\arg a|,|\arg b|<\pi . B(\alpha, \beta)$ is Euler's beta function.) We can easily show

$$
\mathcal{I}_{n}(s)=3^{-s} e^{\frac{\pi i}{2}(2 s-1)} \frac{\Gamma(n+2 s-1)}{\Gamma(s) \Gamma(n+s)} F\left(1-s, s ; n+s ; \frac{2}{3}\right),
$$

and especially $\mathcal{I}_{0}^{\prime}(0)=\frac{3}{2} i, \mathcal{I}_{1}^{\prime}(0)=\frac{\pi}{2}$. Putting $($ C.10 $\sim($ C.16) and C.18 together, we obtain

$$
\zeta_{0}^{\prime}(0)=-\ln \left(E(x)+\frac{2 x(1-x)}{1+x}\right)-\ln \frac{2(1+x)}{(1-x)^{3}}+\frac{1}{2} \ln \frac{\sqrt{-x}}{\pi(1-x)} .
$$

Next we define

$$
X(s)=\sum_{N=0}^{\infty}\left[\left(\lambda_{N 0}-1\right)\left(\lambda_{N 0}+2\right)\right]^{-s} \alpha_{N}^{2},
$$




$$
\begin{gathered}
Y(s)=\sum_{N=0}^{\infty}\left[\left(\lambda_{N 0}-1\right)\left(\lambda_{N 0}+2\right)\right]^{-s} \alpha_{N} \beta_{N}, \\
Z(s)=\sum_{N=0}^{\infty}\left[\left(\lambda_{N 0}-1\right)\left(\lambda_{N 0}+2\right)\right]^{-s} \beta_{N}^{2} .
\end{gathered}
$$

After analytic continuation, these functions at $s=1$ will provide regularized values of $X, Y$ and $Z$ defined in (3.22). To rewrite $(\mathrm{C} .20) \sim(\mathrm{C} .22$ ) as contour integrals we have to analytically continue $\mathcal{N}_{N 0}$. This will be done in terms of the relation

$$
\begin{aligned}
4 \pi\left(\mathcal{N}_{\lambda}\right)^{-2}= & \int_{\cos \theta_{M}}^{1} d w\left(P_{\lambda}(w)\right)^{2} \\
= & \frac{1}{2 \lambda+1}\left[P_{\lambda}\left(\cos \theta_{M}\right) P_{\lambda-1}\left(\cos \theta_{M}\right)+\lambda P_{\lambda}\left(\cos \theta_{M}\right) \frac{\partial}{\partial \lambda} P_{\lambda-1}\left(\cos \theta_{M}\right)\right. \\
& \left.-\lambda P_{\lambda-1}\left(\cos \theta_{M}\right) \frac{\partial}{\partial \lambda} P_{\lambda}\left(\cos \theta_{M}\right)-\cos \theta_{M}\left\{P_{\lambda}\left(\cos \theta_{M}\right)\right\}^{2}\right] .
\end{aligned}
$$

In the last equality use is made of a formula tabulated in Vol I, Chapter III, 3.12 of [24]. A phase of $\mathcal{N}_{\lambda}$ is chosen to be positive when $\lambda$ is a real number. The functions (C.20) (C.22) are now written as

$$
\begin{aligned}
X(s) & =\int_{\gamma_{1}} \frac{d \lambda}{2 \pi i}[(\lambda-1)(\lambda+2)]^{-s}(\alpha(\lambda))^{2} \frac{\partial}{\partial \lambda} \ln G_{0}(\lambda ; x), \\
Y(s) & =\int_{\gamma_{1}} \frac{d \lambda}{2 \pi i}[(\lambda-1)(\lambda+2)]^{-s} \alpha(\lambda) \beta(\lambda) \frac{\partial}{\partial \lambda} \ln G_{0}(\lambda ; x), \\
Z(s) & =\int_{\gamma_{1}} \frac{d \lambda}{2 \pi i}[(\lambda-1)(\lambda+2)]^{-s}(\beta(\lambda))^{2} \frac{\partial}{\partial \lambda} \ln G_{0}(\lambda ; x),
\end{aligned}
$$

where

$$
\alpha(\lambda)=\frac{y}{-x} \mathcal{N}_{\lambda} \frac{\sin \theta_{M}}{\lambda(\lambda+1)} P_{\lambda}^{1}\left(\cos \theta_{M}\right), \quad \beta(\lambda)=\frac{\sqrt{y}}{1-x} \mathcal{N}_{\lambda} P_{\lambda}\left(\cos \theta_{M}\right)
$$

An asymptotic form of $\mathcal{N}_{\lambda}$ for $\operatorname{Im} \lambda \rightarrow+\infty$ is found by using (C.8)

$$
\mathcal{N}_{\lambda} \sim 4 \pi \lambda e^{i\left(\lambda+\frac{1}{2}\right) \theta_{M}+\frac{\pi}{2} i}
$$

By using the recursion formula 20

$$
P_{\lambda}^{1}(\cos \theta)=\frac{\lambda}{\sin \theta}\left[\cos \theta P_{\lambda}(\cos \theta)-P_{\lambda-1}(\cos \theta)\right],
$$

the following asymptotic forms in the same limit are also obtained.

$$
\alpha(\lambda) \sim e^{\frac{\pi}{4} i} \frac{2 y}{(1-x) \sqrt{-x}} \sqrt{\frac{8 \pi}{\lambda \sin \theta_{M}}}, \quad \beta(\lambda) \sim e^{\frac{3}{4} \pi i} \frac{\sqrt{y}}{1-x} \sqrt{\frac{8 \pi \lambda}{\sin \theta_{M}}} .
$$

Now the contour $\gamma_{1}$ can be shifted to $\gamma_{2}$ as in ( C.6. $)$ and the integrals will be reduced to those of discontinuity along the cut. Let us first consider

$$
X(s)=\frac{\sin \pi s}{\pi} e^{\frac{\pi}{2} i s} \int_{0}^{\infty} d t t^{-s}(3+i t)^{-s}(\alpha(1+i t))^{2} \frac{\partial}{\partial t} \ln G_{0}(1+i t ; x) .
$$


This is defined for $0<\operatorname{Re} s<1$. By integrating $X(s)$ by parts by using $t^{-s}=\frac{1}{1-s} \frac{\partial}{\partial t} t^{1-s}$, we obtain

$$
X(s)=\frac{\sin \pi s}{\pi(s-1)} e^{\frac{\pi}{2} i s} \int_{0}^{\infty} d t t^{1-s} \frac{\partial}{\partial t}\left\{(3+i t)^{-s}(\alpha(1+i t))^{2} \frac{\partial}{\partial t} \ln G_{0}(1+i t ; x)\right\} .
$$

As $s \rightarrow 1-0$ this yields

$$
X(1)=\left.\left[-\frac{1}{3}[\alpha(\lambda)]^{2} \frac{\partial}{\partial \lambda} \ln G_{0}(\lambda ; x)\right]\right|_{\lambda=1} .
$$

Similarly the integral for $Y(s)$ is defined for $\frac{1}{2}<\operatorname{Re} s<1$ and we obtain

$$
Y(1)=\left.\left[-\frac{1}{3} \alpha(\lambda) \beta(\lambda) \frac{\partial}{\partial \lambda} \ln G_{0}(\lambda ; x)\right]\right|_{\lambda=1}
$$

Finally $Z(s)$ is slightly complicated. We first decompose the contour integral along $\gamma_{2}$ into three terms and then rewrite them as integrals along the cut. They are given by

$$
\begin{gathered}
Z^{(1)}(s)=\frac{\sin \pi s}{\pi} e^{\frac{\pi}{2} i s} \int_{0}^{\infty} d t t^{-s}(3+i t)^{-s}(\beta(1+i t))^{2}\left[\frac{\partial}{\partial t} \ln G_{0}(1+i t ; x)-\theta_{M}\right] \\
Z^{(2)}(s)=\frac{\sin \pi s}{\pi} e^{\frac{\pi}{2} i s} \theta_{M} \int_{0}^{\infty} d t t^{-s}(3+i t)^{-s}\left[(\beta(1+i t))^{2}-(\beta(1))^{2}\right] \\
Z^{(3)}(s)=\frac{\sin \pi s}{\pi} e^{\frac{\pi}{2} i s} \theta_{M}(\beta(1))^{2} \int_{0}^{\infty} d t t^{-s}(3+i t)^{-s}
\end{gathered}
$$

The first term, which is valid for $\frac{1}{2}<\operatorname{Re} s<1$, can be evaluated at $s \rightarrow 1-0$ as in (C.33).

$$
Z^{(1)}(1)=\left.\left[-\frac{1}{3}[\beta(\lambda)]^{2} \frac{\partial}{\partial \lambda} \ln G_{0}(\lambda ; x)\right]\right|_{\lambda=1}-\frac{i}{3} \theta_{M}[\beta(1)]^{2}
$$

The second one is valid for $1<\operatorname{Re} s<2$ and partial integration yields

$$
\begin{aligned}
Z^{(2)}(s) & =\frac{\sin \pi s}{\pi(s-1)} e^{\frac{\pi}{2} i s} \theta_{M} \int_{0}^{\infty} d t t^{1-s} \frac{\partial}{\partial t}\left[(3+i t)^{-s}\left\{(\beta(1+i t))^{2}-(\beta(1))^{2}\right\}\right] \\
\underset{s \rightarrow 1+0}{\longrightarrow} & -\frac{4 \pi y}{(1-x) \sqrt{-x}} \theta_{M} .
\end{aligned}
$$

The third one can be evaluated by using (C.16),

$$
Z^{(3)}(1)=\theta_{M}[\beta(1)]^{2} \mathcal{I}_{0}(1)=\frac{i}{3} \theta_{M}[\beta(1)]^{2},
$$

which together with C.38 $) \sim($ C.39 $)$ gives

$$
Z(1)=\left.\left[-\frac{1}{3}[\beta(\lambda)]^{2} \frac{\partial}{\partial \lambda} \ln G_{0}(\lambda ; x)\right]\right|_{\lambda=1}-\frac{4 \pi y}{(1-x) \sqrt{-x}} \theta_{M}
$$

By combining (C.33), (C.34) and (C.41) we obtain

$$
X(1) Z(1)-Y(1)^{2}=\left.\frac{4 \pi y}{3(1-x) \sqrt{-x}} \theta_{M}[\alpha(1)]^{2}\left[\frac{\partial}{\partial \lambda} \ln G_{0}(\lambda ; x)\right]\right|_{\lambda=1}
$$


Using

$$
\mathcal{N}_{1}=\sqrt{\frac{-6 \pi(1-x)^{3}}{x\left(3+x^{2}\right)}}, \quad \alpha(1)=\frac{y \sqrt{48 \pi}}{\sqrt{-x(1-x)\left(3+x^{2}\right)}}
$$

and

$$
\left.\left[\frac{\partial}{\partial \lambda} \ln G_{0}(\lambda ; x)\right]\right|_{\lambda=1}=\frac{2 x}{1+x} \frac{E(x)+\frac{1}{2}(1-x)(3+x)}{E(x)+\frac{2 x(1-x)}{1+x}}+\ln \frac{1}{1-x},
$$

which follows 20] from

$$
\left.\frac{\partial}{\partial \lambda} P_{\lambda}(w)\right|_{\lambda=1}=w-1+w \ln \frac{1+w}{2}
$$

we obtain

$$
X Z-Y^{2}=\frac{64 \pi^{2} y^{3} \theta_{M}}{(-x)^{3 / 2}(1-x)^{2}\left(3+x^{2}\right)}\left[\frac{2 x}{1+x} \frac{E(x)+\frac{1}{2}(1-x)(3+x)}{E(x)+\frac{2 x(1-x)}{1+x}}+\ln \frac{1}{1-x}\right] .
$$

By combining this with $(\mathrm{C.2})$ and $(\mathrm{C.19})$ we have

$$
\begin{gathered}
\hat{I}=\quad \frac{(-x)^{7 / 8}(1-x)^{9 / 4}}{2 y^{3 / 2}} \sqrt{\frac{3+x^{2}}{(4 \pi)^{5 / 2} \theta_{M}}}\left[2 x\left(E(x)+\frac{1}{2}(1-x)(3+x)\right)\right. \\
\left.+(1+x)\left(E(x)+\frac{2 x(1-x)}{1+x}\right) \ln \frac{1}{1-x}\right]^{-\frac{1}{2}}
\end{gathered}
$$

Zeta function for $\lambda_{N m}(m \geq 1)$

We define functions

$$
\begin{gathered}
\zeta_{1}(s)=2 \sum_{N=2}^{\infty}\left(\lambda_{N 1}-1\right)^{-s}\left(\lambda_{N 1}+2\right)^{-s} \\
\zeta(s)=2 \sum_{m=2}^{\infty} \sum_{N=m}^{\infty}\left(\lambda_{N m}-1\right)^{-s}\left(\lambda_{N m}+2\right)^{-s}
\end{gathered}
$$

and rewrite them as

$$
\begin{gathered}
\zeta_{1}(s)=2 \int_{\gamma_{1}} \frac{d \lambda}{2 \pi i}[(\lambda-1)(\lambda+2)]^{-s} \frac{\partial}{\partial \lambda} \ln G_{1}(\lambda ; x), \\
\zeta(s)=2 \sum_{m=2}^{\infty} \int_{\gamma_{1}} \frac{d \lambda}{2 \pi i}[(\lambda-1)(\lambda+2)]^{-s} \frac{\partial}{\partial \lambda} \ln G_{m}(\lambda ; x) .
\end{gathered}
$$

The functions $G_{m}$ are defined by

$$
\begin{gathered}
G_{1}(\lambda ; x)=\frac{\Gamma(\lambda-1)}{\Gamma(\lambda+3)}\left[(\lambda-1) \frac{1+x}{1-x} P_{\lambda}^{1}\left(\frac{1+x}{1-x}\right)-(\lambda+1) P_{\lambda-1}^{1}\left(\frac{1+x}{1-x}\right)\right], \\
G_{m}(\lambda ; x)=(-1)^{m} \frac{\Gamma(\lambda-m+1)}{\Gamma(\lambda+m+1)}\left[(\lambda-1) \frac{1+x}{1-x} P_{\lambda}^{m}\left(\frac{1+x}{1-x}\right)-(\lambda+m) P_{\lambda-1}^{m}\left(\frac{1+x}{1-x}\right)\right]
\end{gathered}
$$


$(m \geq 2)$.

The contour $\gamma_{1}$ encircles counterclockwise $\lambda_{N, 1}(N \geq 2)$ and $\lambda_{N, m}(N \geq m)$, respectively, as in fig.1. The contour will then be shifted to $\gamma_{2}$.

Calculation of $\zeta_{1}^{\prime}(0)$ can be performed by the same technique as in the previous subsection and we obtain

$$
\zeta_{1}^{\prime}(0)=\ln \frac{9(1-x)^{3}}{\pi(-x)^{\frac{5}{2}}(3-x)^{2}} .
$$

To compute $\zeta^{\prime}(0)$ we have to subtract from $\ln G_{m}$ its asymptotic expansion $H_{m}$ for $\lambda \rightarrow$

$1+i \infty$ and $m \rightarrow \infty$ with a ratio $\frac{\lambda}{m}$ fixed, up to order $\frac{1}{m}$, and then add this expansion to $\ln G_{m}-H_{m}$.It turns out that these subtracted terms are more complicated than those for $\zeta_{0}^{\prime}(s), \zeta_{1}^{\prime}(s)$ and we have to perform integration over $\lambda$ and summation over $m$ before setting $s=0$. Evaluation of $\zeta^{\prime}(0)$ is thus left for future investigation.

\section{References}

[1] J. Ambjørn, B. Durhuus and J. Fröhlich, Nucl. Phys. B257 (1985) 433;

J. Ambjørn, B. Durhuus, J. Fröhlich and P. Orland, Nucl. Phys. B270 (1986) 457.

[2] F. David, Nucl. Phys. B257 (1985) 45; B257 (1985) 543.

[3] V. A. Kazakov, I. Kostov and A. A. Migdal, Phys. Lett. 157B (1985) 295.

[4] V. G. Knizhnik, A. M. Polyakov and A. B. Zamolodchikov, Mod. Phys. Lett. A3 (1988) 819.

[5] J. Distler and H. Kawai, Nucl. Phys. B321 (1989) 509;

F. David, Mod. Phys. Lett. A3 (1988) 1651.

[6] M. Goulian and M. Li, Phys. Rev. Lett. 66 (1991) 2051.

[7] R. Nakayama, Phys. Lett. B325 (1994) 347.

[8] J. Ambjørn, J. Jurkiewicz and Y. Makeenko, Phys. Lett. B251 (1990) 517.

[9] G. Moore, N. Seiberg and M. Staudacher, Nucl. Phys. B362 (1991) 665.

[10] J. Ambjørn, L. Chekhov, C. F. Kristjansen and Y. Makeenko, Nucl. Phys. B404 (1993) 127.

[11] H. Kawai, N. Kawamoto, N. Mogami and Y. Watabiki, Phys. Lett. B306 (1993) 19.

[12] J. Ambjørn and Y. Watabiki, Nucl. Phys. B445 (1995) 129.

[13] J. Ambjørn and J. Jurkiewicz, Phys. Lett. B278 (1992) 50.

[14] M. E. Agishtein and A. A. Migdal, Mod. Phys. Lett. A7 (1992) 1039.

[15] J. Ambjørn, J. Jurkiewicz and C. F. Kristjansen, Nucl. Phys. B393 (1993) 601.

[16] A. B. Zamolodchikov, Phys. Lett. 117B (1982) 87.

[17] P. Mansfield and R. Neves, 'Boundary conformal field theories on random surfaces and the non-critical open string', hep-th/9605097. 
[18] O. Alvarez, Nucl. Phys. B216 (1983) 125;

B. Durhuus, P. Olsen and J. L. Petersen, Nucl. Phys. B198 (1982) 157; ibid B201 (1982) 176.

[19] D. B. Ray and I. M. Singer, Adv. Math. 7(1971)145.

[20] Y. S. Gradshtein and M. Ryzhik, 'Table of Integrals, Series and Products'(Academic Press 1994).

[21] M. Bordag, E. Elizalde and K. Kirsten, J. Math. Phys. 37(1996)895;

M. Bordag, B. Geyer, K. Kirsten and E. Elizalde, Commun. Math. Phys. 179 (1996) 215.

[22] M. Abramowitz and I. A. Segun, 'Handbook of Mathematical Functions with Formulas and Mathematical Tables' (Dover Publications, New York 1965).

[23] A. P. Prudnikov, Yu. A. Brychkov and O. I. Marichev, 'Integrals And Series',(Gordon and Breach Science Publishers,New York 1990)

[24] A. Erdélyi, W. Magnus, F. Oberhettinger and F. G. Tricomi, 'Bateman Manuscript Project', Higher Transcendental Functions (McGraw-Hill, New York 1953).

[25] J. Ambørn, K. Hayasaka and R. Nakayama, in preparation.

\section{Figure Captions}

Fig.1 The contours $\gamma_{1}$ and $\gamma_{2}$ in the complex $\lambda$ plane. 


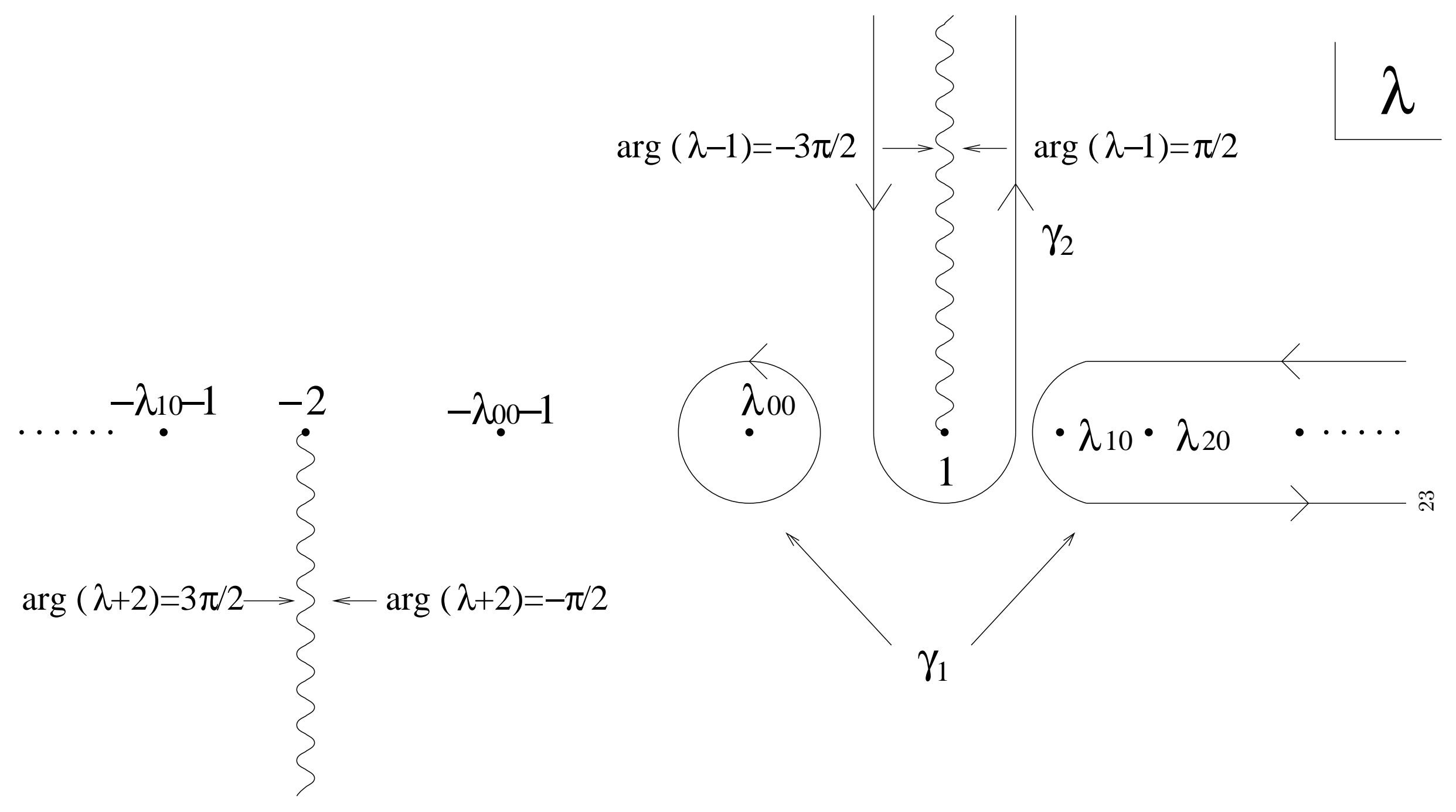

Fig. 1 